\title{
A new insight on postural tachycardia syndrome in 102 adults with hypermobile Ehlers-Danlos Syndrome/hypermobility spectrum disorder
}

\author{
Claudia Celletti ${ }^{1}$, Beatrice Borsellino ${ }^{2}$, Marco Castori ${ }^{3}$, Federica Censi ${ }^{4}$, Giovanni Calcagnini ${ }^{4}$, \\ Filippo Camerota ${ }^{1}$, Stefano Strano ${ }^{2}$
}

${ }^{1}$ Physical Medicine and Rehabilitation, Umberto I University Hospital, Rome; ${ }^{2}$ Department of Heart and Great Vessels "A. Reale", Sapienza University of Rome; ${ }^{3}$ Division of Medical Genetics, Fondazione IRCCS-Casa Sollievo della Sofferenza, San Giovanni Rotondo (FG); ${ }^{4}$ Department of Technology and Health, Italian Institute of Health, Rome, Italy

\begin{abstract}
There is an association between joint hypermobility, hypermobile Ehlers-Danlos syndrome (hEDS) and different forms of orthostatic intolerance. Objective: to explore autonomic profile in a large cohort of adults with hEDS and hypermobility spectrum disorder (hEDS/HSD) with a multimodal approach. In this observational retrospective study, heart rate, blood pressure and baroreflex sensitivity were estimated in $102 \mathrm{hEDS} / \mathrm{HSD}$ subjects during deep breathing, Valsalva maneuver, standing up: 30-15 ratio, Head-Up Tilt and sustained handgrip. Abnormal results and headup tilt test were common and included postural orthostatic
\end{abstract}

Correspondence: Claudia Celletti, Physical Medicine and Rehabilitation, Umberto I University Hospital, Rome, Italy. E-mail: clacelletti@gmail.com

Keywords: autonomic regulation; hypermobile Ehlers-Danlos syndrome; hypermobility spectrum disorder; orthostatic intolerance.

Contributions: All the authors made a substantive intellectual contribution, performed part of the experiments. All the authors have read and approved the final version of the manuscript and agreed to be accountable for all aspects of the work.

Conflict of interest: The authors declare that they have no competing interests, and all authors confirm accuracy.

Availability of data and materials: The datasets used and/or analyzed during the current study are available from the corresponding author on reasonable request.

Patient consent for publication: Not applicable.

Informed consent: Written informed consent was obtained from a legally authorized representative(s) for anonymized patient information to be published in this article.

Received for publication: 25 March 2020.

Accepted for publication: 9 April 2020.

${ }^{\circ}$ Copyright: the Author(s), 2020

Licensee PAGEPress, Italy

Monaldi Archives for Chest Disease 2020; 90:1286

doi: $10.4081 /$ monaldi.2020.1286

This article is distributed under the terms of the Creative Commons Attribution Noncommercial License (by-nc 4.0) which permits any noncommercial use, distribution, and reproduction in any medium, provided the original author(s) and source are credited. tachycardia syndrome (POTS; 48\%), orthostatic intolerance $(25.5 \%)$ and hypotension (3.9\%). Baroreflex sensitivity was significantly different in individuals with POTS compared to the others. This study confirms the high rate and heterogeneity of abnormal autonomic regulation in hEDS/HSD, and suggests the baroreflex sensitivity might distinguish comorbid POTS from other profiles in this subgroup of patients. Abnormal autonomic regulation is common in adults with hEDS/HSD and should be regularly assessed for tailoring the management approach.

\section{Introduction}

There is a strong association between joint hypermobility, hypermobile Ehlers-Danlos syndrome (hEDS) and different forms of orthostatic intolerance, mostly postural orthostatic tachycardia syndrome [1]. In particular, in the last decade, increasing evidence indicates that a significant proportion of individuals with joint hypermobility syndrome (JHS), which has been dismissed by the current nosology and is now, included within the hypermobility spectrum disorders (HSD) [2], and hEDS present with fatigue and a series of dysautonomic symptoms which significantly affect quality of life of these patients [3]. Treatment measures for these manifestations in this patients' category is currently the same of the general population, but their management remains often unsatisfactorily in the real-world medicine due to the lack of shared recommendations aimed at exploring the heterogeneous pathogenesis of these symptoms in the clinical practice. Accordingly, we previously demonstrated abnormal cardiovascular autonomic profiles and, in particular, a higher baroreflex sensitivity (BRS) in 35 adults with hEDS and JHS [4]. Other research groups in cohorts of adults and children with similar sample sizes [4-8] have obtained overlapping results. Nevertheless, little is known about the clinical applications of this evidence in hEDS/HSD.

The aim of this study was to explore patterns of autonomic regulation in a bigger patients' cohort with hEDS/HSD and to identify possible distinguishing features among the various cardiovascular autonomic patterns in such a heterogeneous phenotypic spectrum.

\section{Patients and Methods}

\section{Population}

Enrolled patients were originally assessed according to Brighton and Villefranche criteria [9,10]. The distinction between 
these two sets of criteria was subsequently attenuated with the introduction of a single phenotypic class termed JHS/hEDS [11]. The new international classification presents stricter criteria for hEDS and introduces a broader phenotype HSD to include all symptomatic patients who do not respect the new hEDS criteria [2]. Accordingly, our patients' sample was redefined as hEDS/HSD. Patients recruited have all symptoms related to orthostatic intolerance.

\section{Examination}

Following preliminary evidence, all patients underwent assessment by an autonomic cardiovascular lab - syncope unit as part of the routine multispecialty evaluation for adults HSD/hEDS. ECG and blood pressure signals were noninvasively acquired by using the Task Force Monitor (CNSystem). Heart rate variation, blood pressure response and baroreflex sensitivity (BRS) were estimated by the device software. Tests included Cardiovascular Reflex Tests (deep breathing, Valsalva maneuver, standing up: 3015 ratio, blood pressure response to sustained handgrip), and Head-Up Tilt test.

An expert consensus statement defines Postural Orthostatic Tachycardia Syndrome (POTS), a form of chronic orthostatic intolerance, as a heart rate increment $\geq 30$ beats/min within $10 \mathrm{~min}$ of standing or head-up-tilt (HUT) which is sustained in the absence of orthostatic hypotension (a drop $>20 \mathrm{mmHg}$ in systolic blood pressure or $>10 \mathrm{mmHg}$ in diastolic body pressure) [12].

\section{Deep breathing}

Participants breathed maximally at a frequency of 6 breaths per minute, following the lead of an oscillating ball on a computer screen. The deep breathing test was considered normal if heart rate (HR) variation was 15 beats/min or more, borderline if 11-14 beats/min, and pathological if 10 beats/min or less. The HR range was calculated as a measure of parasympathetic reactivity [6].

\section{Valsalva maneuver}

Individuals blew into a mouthpiece between 40 and $50 \mathrm{mmHg}$ for $15 \mathrm{~s}$. The Valsalva ratio (VR) was calculated, and the 4 phases of blood pressure (BP) response were quantified [13]. Respect to the baseline, the maximal drop of systolic and diastolic BP (SBP and DBP) at the different phases during Valsalva maneuver were computed.

\section{Standing up}

Subjects were asked to stand at rest in the supine position for $5 \mathrm{~min}$ and, then, to assume the standing position without help as quickly as possible according to his/her physical abilities. The cardiac chronotropic response characteristic is expressed by the ratio 30:15; this ratio means the longest $R-R$ interval after the maneuver (around the thirtieth beat) and the shortest R-R interval around the fifteenth beat. Results were evaluated considering as normal a ratio $\geq 1.04$, as borderline when the ratio value was between 1.01 and 1.03, and as pathological when the value is $\leq 1.00[4]$.

\section{Sustained handgrip}

Handgrip was maintained at $30 \%$ of maximal voluntary contraction until the maximum time of $5 \mathrm{~min}$ by using a dynamometer. Handgrip test was considered successfully performed if patients were able to maintain a constant effort for at least $1 \mathrm{~min}$.

\section{Head-up tilt}

The participant firstly rested in supine position quietly for 5 min. Baseline HR and BP were calculated as the mean from $40 \mathrm{~s}$ to $10 \mathrm{~s}$ before tilting. Next, the table was tilted upright to an angle of $70^{\circ}$ for a maximum of $20 \mathrm{~min}$. Orthostatic hypotension $(\mathrm{OH})$ was defined as a sustained diastolic BP drop of at least $10 \mathrm{mmHg}$ or a systolic BP drop of at least $20 \mathrm{mmHg}$ [13]. Postural orthostatic tachycardia (POTS) was defined as a sustained HR rise of at least $30 \mathrm{bpm}$ or a HR of at least $120 \mathrm{bpm}$ in the first $10 \mathrm{~min}$ of tilt, without concomitant orthostatic hypotension [14].

BRS was assessed using the sequence method in which "up" and "down" sequences are identified (CNSystems Task-Force Monitor 3040i). Up sequences consist of four or more consecutive cardiac cycles for which there is a sequential rise in both systolic pressure and R-R interval. Down sequences consist of four of more cardiac cycles for which there is a sequential fall in systolic pressure and R-R interval. Values of cardiac BRS were accepted when the number of sequences was $\geq 3$ for both up and down sequences $[14,15]$.

\section{Statistical analysis}

Student's paired $t$-test was used to compare differences in the spectral variables between rest and tilt. Student's unpaired $t$-test was used to compare differences in the spectral variables between groups. A p-value of $<0.01$ was considered to indicate statistical significance.

\section{Results}

A total of $102 \mathrm{hEDS} / \mathrm{HSD}$ individuals (90 females and 12 males, mean age $34 \pm 13$ ) were evaluated. Among the group about $48 \%$ of the patients refereed syncopal and pre-syncopal symptoms, $18.6 \%$ complained fatigue, $16.6 \%$ tachycardia, $8.8 \%$ vertigo and $7.8 \%$ cephalalgia. Table 1 shows that DB test, $30 / 15$ ratio and VR gave normal results in most patients. All subjects failed to complete the sustained handgrip test due to pain and fatigue. Seventy-five individuals $(73.5 \%)$ were able to perform the Valsalva maneuver, while the remaining $(27.5 \%)$ were not due to breathing difficulties and incoordination. In those who completed the Valsalva maneuver, we observed both the increase of blood pressure (BP) between early and late phase II, and the overshoot in late phase IV, according to the normal values (Table 2).

Ninety-nine out of 102 subjects completed the HUT test. Among them, 49 (49.49\%) showed POTS, 26 (26.26\%) orthostatic intolerance and $4(4.04 \%)$ orthostatic hypotension, while the remaining (20.20\%) showed a normal response. Percentages about heart rate (HR) and BP for each categories are comparable to the previous study with a net predominance of POTS (48\%) and orthostatic intolerance $(25.5 \%)$ in the patients' sample (Table 3 ).

The BRS values were estimated and compared at rest and during the HUT test in the two POTS hEDS/HSD (individuals with POTS at HUT test) versus non-POTS-hEDS/HSD (individuals without POTS at HUT test) and POTS hEDS/HSD versus Normal subjects (Table 4). Subjects with orthostatic hypotension at HUT were excluded from this analysis.

At rest, POTS hEDS/HSD subjects showed higher BRS values compared to non-POTS hEDS/HSD $(\mathrm{p}<0.03)$ and normalhEDS/HSD $(\mathrm{p}<0.009)$ subjects. 
Table 1. Response to cardiovascular reflex tests.

\begin{tabular}{lcccc} 
Test (Total \#102) & Normal number (\%) & Borderline number (\%) & Pathological number (\%) & Not evaluable number (\%) \\
Deep breathing & $100(98)$ & - & - & $2(1.9)$ \\
Valsalva ratio & $70(68.6)$ & $5(4.9)$ & $1(0.9)$ & $27(26.4)$ \\
\hline $30 / 15$ ratio & $80(78.4)$ & $20(19.6)$ & - & $1(0.9)$ \\
Sustained handgrip & - & - & $102(100)$ & \\
\hline
\end{tabular}

Table 2. Results of Valsalva maneuver in the 75 hEDS/HSD individuals.

\begin{tabular}{lc} 
Variable & $\mathrm{mmHg} \pm \mathrm{SD}$ \\
Baseline SBP/DBP & $111.5 \pm 10.9$ \\
Maximal drop of SBP during early phase II & $71.6 \pm 8.1$ \\
\hline Maximal drop of DBP during early phase II & $10.7 \pm 14.6$ \\
SBP at late phase II & $114.3 \pm 18.3$ \\
\hline DBP at late phase II & $81.1 \pm 14.5$ \\
SBP at late phase IV & $126.3 \pm 20.2$ \\
\hline DBP at late phase IV & $79 \pm 13.7$ \\
Difference between late phase II SBP and early phase II SBP & $24.7 \pm 13.6$ \\
\hline Difference between late phase II DBP and early phase II DBP & $14.7 \pm 9.5$
\end{tabular}

Normal values: Maximal drop of mean blood pressure (MBP) during early phase II: $\geq 20 \mathrm{mmHg}$; systolic blood pressure (SBP) and diastolic blood pressure (DBP) at late phase II: $\geq$ baseline; SBP and DBP at late phase IV: $\geq$ baseline.

Table 3. Heart rate (HR) and blood pressure responses after head-up tilt test.

\begin{tabular}{|c|c|c|c|c|c|c|}
\hline $\begin{array}{l}\text { Phenotype } \\
\text { (Total \#102) }\end{array}$ & $\begin{array}{c}\text { HR response } \\
\text { ratio (\%) }\end{array}$ & $\begin{array}{l}\text { HR mean } \\
\text { difference } \\
\text { (bpm } \pm \text { SD) }\end{array}$ & & $\begin{array}{c}\text { BP response } \\
\text { ratio (\%) }\end{array}$ & $\begin{array}{c}\text { SBP mean } \\
\text { difference } \\
(\mathrm{mmHg} \pm \mathrm{SD})\end{array}$ & $\begin{array}{l}\text { DBP mean } \\
\text { difference } \\
(\mathrm{mmHg} \pm \mathrm{SD})\end{array}$ \\
\hline $\begin{array}{l}\text { Normal } \\
\text { (HR increase between } \\
10 \text { and } 19 \mathrm{bpm} \text { ) }\end{array}$ & $20(19.6)$ & $12.5 \pm 4.3$ & Normal & $96(94.1)$ & $6.5 \pm 6.1$ & $6.7 \pm 5.5$ \\
\hline POTS(HR increase $>30 \mathrm{bpm}$ ) & $49(48)$ & $37 \pm 6.3$ & & & & \\
\hline $\begin{array}{l}\text { Reduced orthostatism } \\
\text { tolerance (HR increase from } \\
20 \text { to } 29 \mathrm{bpm} \text { ) }\end{array}$ & $26(25.5)$ & $23.5 \pm 3$ & $\begin{array}{l}\text { Orthostatic hypotension } \\
\text { (reduced peripheral } \\
\text { vessel response) }\end{array}$ & $3(2.9)$ & $-2.5 \pm 21$ & $-16.2 \pm 2.5$ \\
\hline $\begin{array}{l}\text { Orthostatic hypotension } \\
\text { (reduced peripheral vessel } \\
\text { response) }\end{array}$ & $4(3.9)$ & $28 \pm 2.6$ & & & & \\
\hline Not evaluable & $3(2.9)$ & - & Not evaluable & $3(2.9)$ & - & - \\
\hline
\end{tabular}

Table 4. Comparison of baroreflex sensitivity between hEDS/EDS individuals with and without POTS and between hEDS/EDS individuals with POTS and hEDS/HSD individuals with normal results at head-up tilt test.

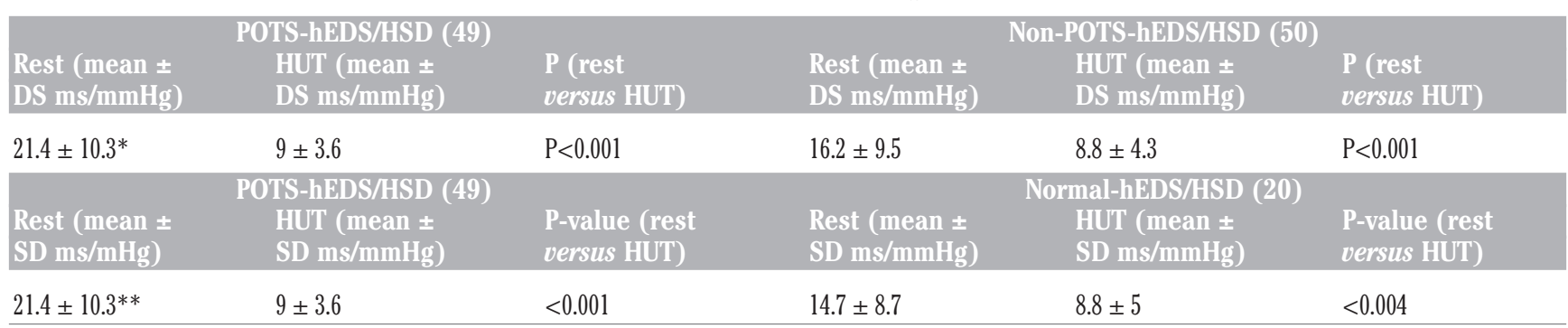

${ }^{*} \mathrm{p}<0.03$ POTS us non-POTS; ${ }^{* *} \mathrm{p}<0.009$ POTS us normal. 


\section{Discussion}

These findings confirm, in the largest patients' cohort published to date, that $\sim 50 \%$ of adults with hEDS/HSD present comorbid POTS and that a higher proportion of them shows an abnormal response at HUT test. As POTS and hEDS/HSD individuals share many chronic symptoms that might be ameliorated by treating the former, our study suggests testing hEDS/HSD individuals for POTS in a clinical setting for approaching a more tailored management program. Accordingly, a list of useful treatment resources for fatigue and cardiovascular dysautonomia have been recently reviewed for Ehlers-Danlos syndrome and related disorders $[16,17]$.

Our study also proposes that, among adults with hEDS/HSD, those with POTS might be distinguished from the others by BRS estimated with sequences method during HUT. Therefore, we speculate that BRS indirect estimation in basal conditions could be considered an alternative for separating hEDS/HSD individuals with comorbid POTS from the others in the absence of accessibility to HUT test. If confirmed by further studies and/or in other populations, HUT test and BRS indirect estimation might be included, in combination or isolation, in future management guidelines for optimal treatment of cardiovascular dysautonomic manifestations in hEDS/HSD patients/adults, in association to management and rehabilitation strategy related to muscle hypotonia $[18,19]$ in order to improve balance, stability and reduce cardiovascular dysautonomia.

There are some limitation in this study: firstly this is a retrospective evaluation and in a future a more organized study should be desirable; moreover a correlation between different symptoms and their intensity and, duration of the disease and possible other pathology or therapy should be useful.

\section{Conclusions}

In conclusion, this study present cardiovascular autonomic data in the largest patients' cohort with HSD/hEDS published to date. We confirmed the high frequency of POTS and orthostatic intolerance in $\mathrm{HSD} / \mathrm{hEDS}$ and their potential relevance in the clinical assessment of these patients. The existence of different autonomic profiles in our sample suggests discrete phenotypes within the HSD/hEDS spectrum. Further exploration of such heterogeneity may help in the identification of biomarkers useful for both clinical and molecular research.

\section{References}

1. Roma M, Marden CL, De Wandele I, et al. Postural tachycardia syndrome and other forms of orthostatic intolerance in EhlersDanlos syndrome. Auton Neurosci 2018;215:89-96.

2. Malfait F, Francomano C, Byers P. The 2017 international classification of the Ehlers-Danlos syndromes. Am J Med Genet C Semin Med Genet 2017;175:8-26.

3. Copetti M, Morlino S, Colombi M, et al. Severity classes in adults with hypermobile Ehlers-Danlos syndrome/hypermobility spectrum disorders: a pilot study of 105 Italian patients. Rheumatology (Oxford) 2019;58:1722-30.

4. Celletti C, Camerota F, Castori M, et al. Orthostatic intolerance and postural orthostatic tachycardia syndrome in joint hypermobility syndrome/Ehlers-Danlos syndrome, hypermobility type: neurovegetative dysregulation or autonomic failure? Biomed Res Int 2017;2017:9161865.

5. Rowe PC, Barron DF, Calkins $\mathrm{H}$ et al. Orthostatic intolerance and chronic fatigue syndrome associated with Ehlers Danlos syndrome. J Pediat 1999;135:494-9.

6. De Wandele I, Rombaut L, Leybaert L et al. Dysautonomia and its underlying mechanisms in the hypermobility type of EhlersDanlos syndrome. Semin Arthritis Rheum 2014;44:93-100.

7. Wallman D, Weinberg J, Hohler AD. Ehlers-Danlos syndrome and postural tachycardia syndrome: a relationship study. J Neurol Sci 2014;340:99-102.

8. Cheng JL, Au JS, Guzman JC, et al. Cardiovascular profile in postural orthostatic tachycardia syndrome and Ehlers-Danlos syndrome type III. Clin Auton Res 2017;27:113-6.

9. Grahame R, Bird HA, Child A. The revised (Brighton 1998) criteria for the diagnosis of benign joint hypermobility syndrome (BJHS). J Rheumatol 2000;27:1777-9.

10. Beighton P, De Paepe A, Steinmann B, et al. Ehlers-Danlos syndromes: revised nosology, Villefranche, 1997. Ehlers-Danlos National Foundation (USA) and Ehlers-Danlos Support Group (UK). Am J Med Genet 1998;77:31-7.

11. Tinkle BT, Bird HA, Grahame R. The lack of clinical distinction between the hypermobility type of Ehlers-Danlos syndrome and the joint hypermobility syndrome (a.k.a. hypermobility syndrome). Am J Med Genet A 2009;149A:2368-70.

12. Freeman R, Wieling W, Axelrod FB, et al. Consensus statement on the definition of orthostatic hypotension, neurally mediated syncope and the postural tachycardia syndrome. Clin Auton Res 2011;21:69-72.

13. Freeman R, Wieling W, Axelrod FB, et al. Consensus statement on the definition of orthostatic hypotension, neurally mediated syncope and the postural tachycardia syndrome. Auton Neurosci 2011;161:46-8.

14. Bertinieri G, Di Rienzo M, Cavallazzi A, et al. Evaluation of baroreceptor reflex by blood pressure monitoring in unanesthetized cats. Am J Physiol 1988;254:H377-83.

15. Parati G, Di Rienzo M, Bertinieri G. Evaluation of the baroreceptor-heart rate reflex by 24-hour intra-arterial blood pressure monitoring in humans. Hypertension 1988;12:214-22.

16. Hakim A, De Wandele I, O'Callaghan C, et al. Chronic fatigue in Ehlers-Danlos syndrome-Hypermobile type. Am J Med Genet C Semin Med Genet 2017;175:175-80.

17. Hakim A, O'Callaghan C, De Wandele I et al. Cardiovascular autonomic dysfunction in Ehlers-Danlos syndromeHypermobile type. Am J Med Genet C Semin Med Genet 2017;175:168-74.

18. Celletti C, Castori M, Galli M, et al. Evaluation of balance and improvement of proprioception by repetitive muscle vibration in a 15-year-old girl with joint hypermobility syndrome. Arthritis Care Res (Hoboken) 2011;63:775-9.

19. Galli M, Cimolin V, Vismara L, et al. The effects of muscle hypotonia and weakness on balance: a study on Prader-Willi and Ehlers-Danlos syndrome patients. Res Dev Disabil 2011;32:1117-21. 